See discussions, stats, and author profiles for this publication at: https://www.researchgate.net/publication/327150907

\title{
Weaving of Metaheuristics with Cooperative Parallelism
}

Article in Lecture Notes in Computer Science · September 2018

DOI: 10.1007/978-3-319-99253-2_35

CITATIONS

4 authors, including:

Danny Múnera

University of Antioquia

20 PUBLICATIONS 47 CITATIONS

SEE PROFILE

Salvador Abreu

Universidade de Évora

99 PUBLICATIONS 462 CITATIONS

SEE PROFILE

Some of the authors of this publication are also working on these related projects:

Contextual Logic Programming View project

Solving Hard Permutation-based Combinatorial Optimization Problems using Parallel Hybrid Metaheuristics View project
READS

17

Daniel Diaz

Université de Paris 1 Panthéon-Sorbonne

99 PUBLICATIONS 1,519 CITATIONS

SEE PROFILE 


\title{
Weaving of Metaheuristics with Cooperative Parallelism
}

\author{
Jheisson López ${ }^{1,2}$, Danny Múnera ${ }^{2}$, Daniel Diaz $^{3}$, and Salvador Abreu ${ }^{4}$ \\ 1 National University of General Sarmiento, Argentina \\ jalopez@ungs.edu.ar \\ 2 University of Antioquia, Colombia \\ danny.munera@udea.edu.co \\ 3 University of Paris 1/CRI, France \\ daniel.diaz@univ-paris1.fr \\ 4 University of Évora/LISP ${ }^{\star \star}$, Portugal \\ spa@uevora.pt
}

\begin{abstract}
We propose PHYSH (Parallel HYbridization for Simple Heuristics), a framework to ease the design and implementation of hybrid metaheuristics via cooperative parallelism. With this framework, the user only needs encode each of the desired metaheuristics and may rely on PHYSH for parallelization, cooperation and hybridization. PHYSH supports the combination of population-based and single-solution metaheuristics and enables the user to control the tradeoff between intensification and diversification. We also provide an open-source implementation of this framework which we use to model the Quadratic Assignment Problem (QAP) with a hybrid solver, combining three metaheuristics. We present experimental evidence that PHYSH brings significant improvements over competing approaches, as witness the performance on representative hard instances of QAP.
\end{abstract}

\section{Introduction}

Metaheuristics are often the most efficient approach to address the hardest Combinatorial Optimization Problems (COP). Metaheuristics are high-level procedures using choices (i.e., heuristics) to limit the part of the search space which actually gets visited, in order to make problems tractable. Metaheuristics can be classified in two main categories: single-solution and population-based methods. Single-solution metaheuristics ( $\mathrm{S}-\mathrm{MH})$ maintain, modify and stepwise improve on a single candidate solution, hence the term trajectory-based metaheuristics. On the other hand, population-based metaheuristics (P-MH), modify and improve a population, i.e. a set of individuals corresponding to candidate solutions.

Metaheuristics generally implement two main search strategies: intensification and diversification, also called exploitation and exploration [1]. Intensification guides the solver to deeply explore a promising part of the search space. In

** This work was partly funded by FCT under grant UID/CEC/4668/2016 (LISP). 
contrast, diversification aims at extending the search onto different parts of the search space [8]. In order to obtain the best performance, a metaheuristic should provide a useful balance between intensification and diversification. By design, some heuristics are good at one but not at the other.

More generally, each metaheuristic can perform differently according to the problem or even instance being solved. A single metaheuristic will also vary depending on its chosen tuning parameters. The current trend is thus to design hybrid metaheuristics, by combining different methods in order to benefit from the individual advantages of each one [9. An effective approach consists in combining an evolutionary algorithm with a single-solution method (very often a local search procedure). These hybrid methods are called memetic algorithms [10. Hybrid metaheuristics tend to be complex procedures, tricky to design, implement and tune, therefore, most of them only combine two methods.

Despite the good results obtained with the use of hybrid metaheuristics, it is still necessary to reduce the processing times needed for harder instances [18. One possible answer entails resorting to parallel execution [5. For instance, several instances of a given metaheuristic can be executed in parallel in order to develop concurrent explorations of the search space, either independently or cooperatively by means of communication between concurrent processes. The first is easiest to implement on parallel computers, as the metaheuristics run oblivious to each other and execution stops as soon as any of them finds a solution 16 22. For some problems this provides very good results [3] but in many cases the speedup tends to taper off when increasing the number of processors [13. A cooperative approach entails adding a communication mechanism in order to share or exchange information among solver instances during the search process [20. However, designing an efficient cooperative method is a dauntingly complex task [4, and many issues must be solved: What information is exchanged? Between which processes is it exchanged? When is it exchanged? How is it exchanged? How is the imported data used? [21. Moreover, most cooperative choices are problem-dependent (and sometimes even instance-dependent). Bad choices result in poor performance, possibly much worse than what could be obtained with independent parallelism. However, a well-tuned cooperative scheme may significantly outperform the independent approach.

In 2014, we proposed the Cooperative Parallel Local Search framework (CPLS) for the cooperative parallel execution of local search metaheuristics [1413]. The user only has to encode the LS procedure and can rely on CPLS to obtain a parallel application able to run concurrently and cooperatively several instances of this LS procedure. At runtime, the outcome is a parallel exploration of the search space with candidate solution interchange. All low-level parallel mechanisms (task creation/destruction, mapping to physical resources, synchronization, communication, termination,...) are transparently handled by CPLS. CPLS has been successfully used to tackle stable matching problems [15 and very difficult instances of the Quadratic Assignment Problem (QAP) [12. We later extended CPLS to allow the user to run different metaheuristics in parallel. CPLS has enabled a simpler way to hybridize metaheuristics, by exploiting its 
solution-sharing cooperative parallelism mechanism. At runtime, the parallel instances of each different metaheuristic communicate their best solutions, and one of them may forgo its current computation to adopt a better solution from the others, hoping to converge faster. The expected outcome is that a solution which may be stagnating for one solver, has a chance to be improved on by another metaheuristic. CPLS has been successfully used to develop a very efficient hybrid solver for QAP [11. However, CPLS was designed for local search metaheuristics: its cooperation mechanisms can only handle single-solution metaheuristics. When pursuing hybridization this limitation becomes too severe.

In this paper we propose a framework for the Parallel HYbridization of Simple Heuristics (PHYSH), which eases the implementation of hybrid metaheuristics using cooperative parallelism. As in CPLS, the user only needs to code each of the desired metaheuristics, independently, and may rely on PHYSH to provide both parallelism and cooperation to get "the best of both worlds". PHYSH is highly parametric and the user has control over the trade-off between intensification and diversification. Single-solutions methods are in charge of intensifying the search while population-based methods can be used to provide diversification through the evolution of a population. We also sketch a prototype implementation, available as an open source library written in the IBM X10 concurrent programming language. needs only code the desired metaheuristic, PHYSH API. We used this implementation to develop a parallel solver for QAP by hybridizing 3 metaheuristics: a Genetic Algorithm, an Extremal Optimization procedure and a Tabu Search method. The resulting solver performs extremely well on the hardest instances of QAP.

The rest of this paper is organized as follows: in section 2 we describe the framework, while in section 3 we discuss implementation issues. In section 4 we carry out an experimental evaluation on hard QAP instances. Finally, we summarize our results and draw plans for future developments in section 5

\section{The PHYSH Framework}

The aim of PHYSH is to offer the user an environment for the development of hybrid and parallel metaheuristics. By transparently managing all of the technical details of parallel programming as well as mechanisms for hybridization, PHYSH allows the user to focus on metaheuristic codings and problem modeling. The resulting parallel hybrid search process starts from different points in the search space, attempting to ensure convergence on proper solutions while escaping local extrema. We achieve this with multiple concurrent worker teams, each one tasked with visiting a different region of the search space. Figure 1 depicts a search space where red regions contain high-quality solutions which is explored by 4 teams in parallel: 2 teams are intensifying the search in a promising region while the 2 others are diversifying the search in order to reach other rich region.

Teams are composed of the following components: a set of search units, a diverse and an elite populations. The main active element of the framework is the search unit (SU) which encapsulates a single metaheuristic that can be 


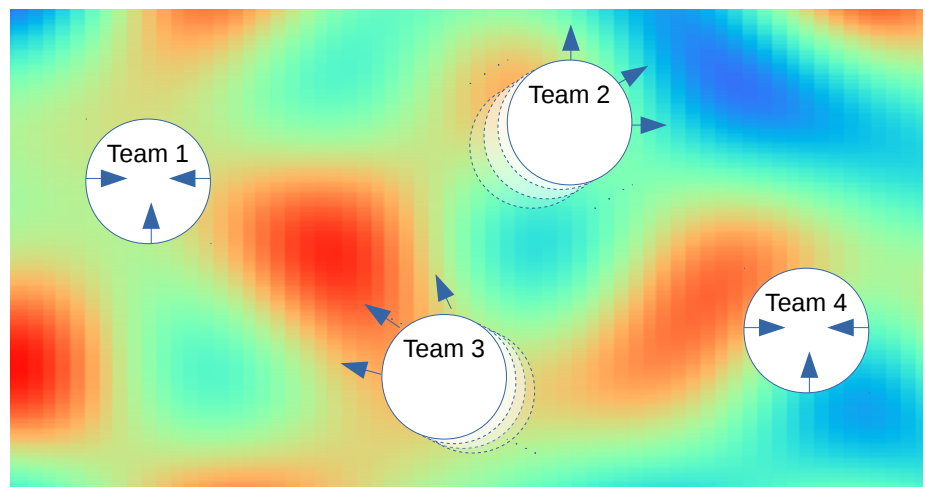

Fig. 1: PHYSH search process

either a S-MH or a $\mathrm{P}-\mathrm{MH}$. If the SU contains a S-MH, it takes the role of an intensifier otherwise (implementing $\mathrm{P}-\mathrm{MH}$ ) it takes the role of a diversifier. The elite population (EP) retains the best individuals found by the intensifiers, while the diverse population (DP) holds individuals sent by diversifiers. The interaction patterns between the different components that make up a team establish a parametric four-way migratory flow process (see Figure 2). In each case a parameter controls the migration frequency 5

- Elite Emigration (ee): from the intensifier worker to the EP.

- Diverse Emigration (de): from the diversifier worker to the DP.

- Elite Immigration (ei): from the EP to the diversifier worker.

- Diverse Immigration (di): from the DP to the intensifier worker.

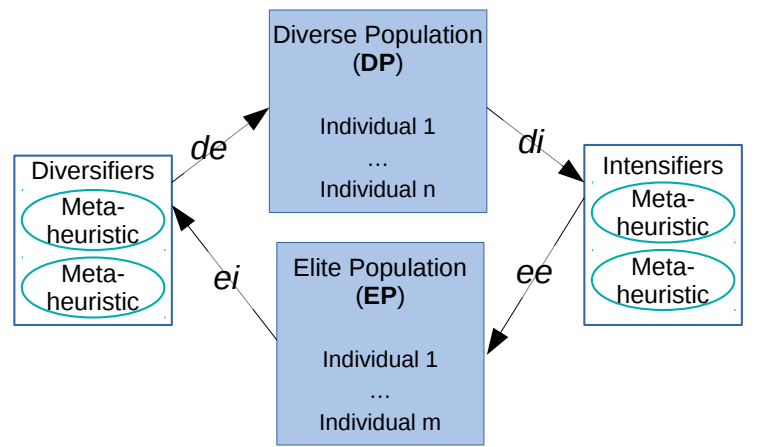

Fig. 2: PHYSH team structure

The intensifiers (resp. diversifiers) must apply a selection policy to determine which individuals emigrate to the EP (resp. DP). EP and DP population implement an acceptance policy for deciding whether the incoming individual is

\footnotetext{
5 Terms "immigration" and "emigration" are from the metaheuristics point-of-view.
} 
accepted or rejected (discarded). For immigration flows, intensifiers and diversifiers request individuals respectively from DP and EP. Once again a selection policy is implemented on the populations to define how to chose an individual and send it to the corresponding entity.

Our framework follows the design principle of separating policy form mechanism. As a result, this process constitutes a flexible interaction model between intensifiers and diversifiers which eases the hybridization of simple metaheuristics, effectively promoting cross-fertilization among different types.

Different mechanisms can be implemented for the same policy e.g., an elitist or non-elitist mechanism. In the first case we favor elite individuals, while in the second we may, for instance, select the most diverse individual or even adopt a stochastic stance. We may assign several mechanisms for the same policy to a component, in that case the mechanisms are applied in a round-robin fashion until they succeed in the (selection/acceptance) pipeline.

An intuitive configuration could assign elitist mechanism to the intensifiers, non-elitist mechanism to the diversifiers, and both types of mechanism to the populations. We decided to make this a configurable option, as it provides rich choices of search strategy.

In PHYSH, the programmer may easily control the balance between intensification and diversification (see Figure 3). Take the proportion of SUs used for the intensifiers vs. diversifiers: it may be tuned to achieve a specific balance. For instance, if more intensification is needed for a given instance, one may increase the number of SUs in the role of intensifier. The intensification/diversification level may also be tweaked by varying the number of teams in the execution: given a fixed number of processing units, using more teams with a lower $\mathrm{SU}$ count will increase the diversification on the search.

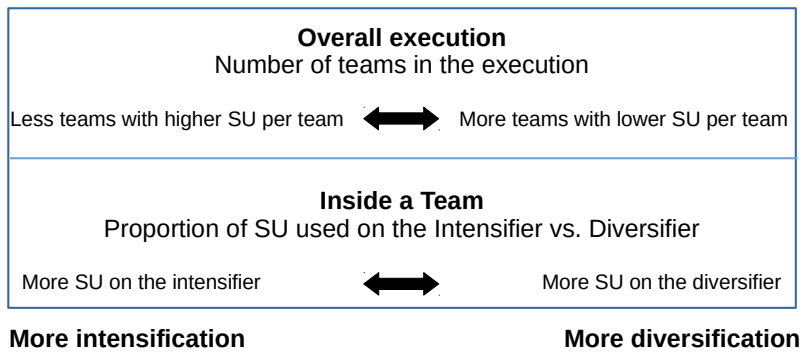

Fig. 3: PHYSH Intensification-Diversification control

The PHYSH framework is designed to adapt to different parallel architectures: shared-memory multiprocessors as well as distributed systems with network-connected MP nodes. SUs are meant to be mapped to physical processors, while teams may be configured very flexibly. 


\section{PHYSH $\times$ 10: a Prototype Implementation}

We implemented our prototype in the X10 programming language which is a high level object-oriented programming language, focused on concurrency and distribution. X10 supports a wide range of parallel platforms and it has been in active developemnt by IBM research since 2004. X10 is based on the Asyncronous Partitioned Global Address Space model (APGAS). Using this model, computation and data are partitioned into places which are abstractions for mutable, shared-memory regions that can contain global references to locations in other places, as well as worker threads operating on this memory.

In adoption of common practice for metaheuristics tools, $\mathrm{PHYSH} \times 10$ presents a clear separation between available metaheuristics and the problems that can be solved. We have implemented a genetic algorithm (GA), a robust tabu search (RoTS) and an extremal optimization (EO) procedure. Consequently, the diversifiers are built from SUs that contain a GA, while the other two metaheuristics are available for the SUs in the intensifiers. Figure 4 displays the main classes of $\mathrm{PHYSH} \times 10$, a few application-specific ones and their relationships.

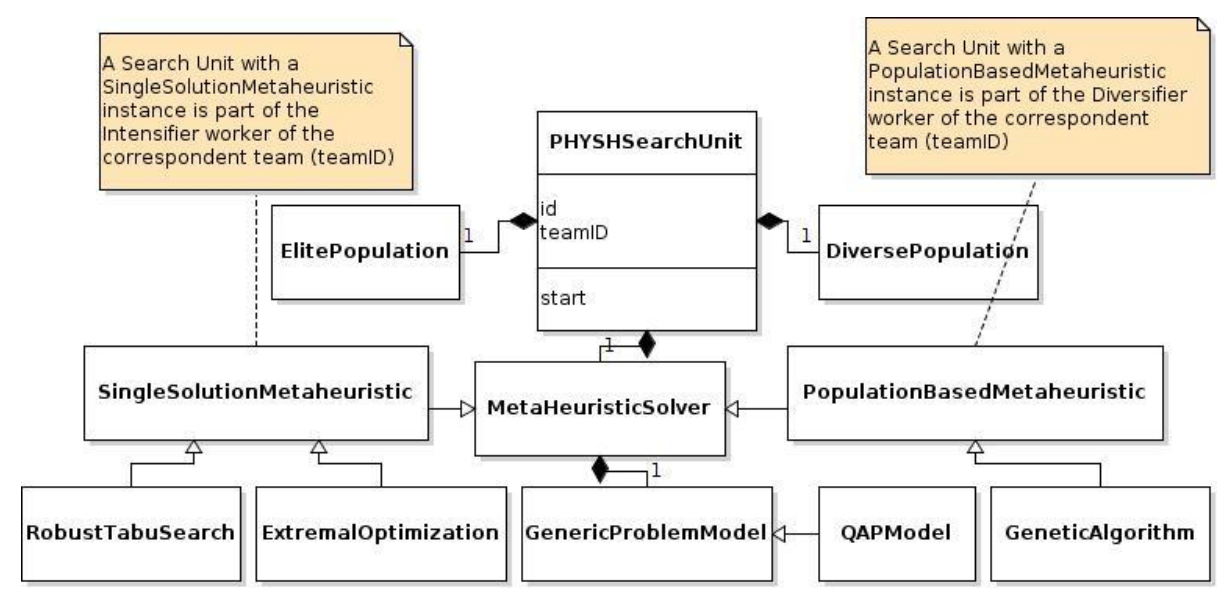

Fig. 4: PHYSH $\times 10$ UML Diagram of the main classes

PHYSH $\times 10$ uses the features offered by X10-APGAS model to assign available physical processing resources. Accordingly, each SU is allocated to an X10 place, so that intensifiers and diversifiers operate as a distributed system. As explain above, SUs are grouped to form teams. Each team is composed of tz SUs. The number of teams is thus \#cores/tz. EP and DP populations are bound to a single SU within each team. These populations have a parametric size i.e., epz individuals for EP and dpz individuals for DP. Each component implements the most convenient mechanism for the acceptance and selection criteria.

At present, $\mathrm{PHYSH} \times 10$ provides the following selection mechanisms:

- Best: best individual found in the search process. 
- Current: all eligible individuals are selected (for S-MH the current configuration is the unique eligible individual.)

- Random: an individual is randomly selected from the elegible set.

The following acceptance mechanism are also provided:

- Elitist: The individual is accepted if it is better than the worst in the target population (if it is not present yet.)

- Probabilistic: The individual is accepted, regardless of its cost, with a given probability (if it is not present yet.)

- Maximizer: The individual is accepted if its average distance to the other individuals is greater than a defined threshold.

Intensifiers implement the current mechanism for the selection policy i.e., SU sends its current configuration to perform the emigration to the EP. Parameter elite emigration period (eep) controls the periodicity of this communication. Intensifiers also request an immigrant individual from DP each diverse immigration period (dip). To accept or deny this individual intensifiers implement an elitist mechanism for the acceptance policy (for S-MH the target "population" is current solution of the metaheuristic).

Diversifiers implements a random mechanism for the selection policy. This mechanism requires a parameter to define the percentage of the population eligible for emigration (ppfe). The individual to emigrate is randomly chosen among the top ppfe\% of the SU's population (the best individuals). Parameter diverse emigration period (dep) controls the periodicity of this emigration process. Diversifiers also request an immigrant from $\mathrm{EP}$ each elite immigration period (eip). Individual diversifiers implement an elitist acceptance mechanism.

To simplify the assignment of these parameters we define two general values: emigration period ep and immigration period ip. Considering teams of size tz (a team embeds $t z$ SUs) and a problem of size of $n$, the default values are computed as follows: eip $=e p / t z, \operatorname{dep}=i p / n$, eep $=e p / n$ and dip $=i p$.

\section{Experimental Evaluation}

To evaluate the performance of our framework, we developed PHYSH-QAP 6 ; a parallel hybrid solver for QAP which combines three metaheuristics: a Genetic Algorithm (GA) [7, a Robust Tabu Search (RoTS) [19] and an Extremal Optimization procedure (EO) 12. PHYSH-QAP is built on top of $\mathrm{PHYSH} \times 10$. We consider three sets of very hard benchmarks: the 33 hardest instances of QAPLIB and two sets of even harder instances: Drezners dre $X X$ and Palubeckis Inst $X X$ instances. All experiments have been carried out on a cluster of 16 machines, each with $4 \times 16$-core AMD Opteron $6376 \mathrm{CPUs}$ running at $2.3 \mathrm{GHz}$ and 128 GB of RAM. The nodes are interconnected with InfiniBand FDR $4 \times$ (i.e., 56 GBPS). We had access to 4 nodes and used up to 32 cores per node.

\footnotetext{
${ }^{6}$ The source code is available from https://github.com/jlopezrf/COPSolver-V_2.0
} 


\subsection{Evaluation of PHYSH-QAP on QAPLIB}

QAPLIB is a collection of 134 QAP problems of different sizes [2]. The instances are generally named as name $X X$ where name corresponds to the first letters of the author and $X X$ is the size of the problem. For each instance, QAPLIB also includes the Best Known Solution (BKS), which is sometimes the optimum. Many QAPLIB instances are easy for a parallel solver, we therefore only considered the 33 hardest instances, as reported in [12]. Each problem instance is executed 30 times, stopping as soon as the BKS is reached or when a time limit of $5 \mathrm{~min}$ is hit, using 64 cores. PHYSH-QAP was configured with four teams, each of size $\mathrm{tz}=16$ embedding 1 diversifier running GA, 8 intensifiers running RoTS and 7 intensifiers running EO. The size for the elite population and the diverse population was set to $4(\mathrm{epz}=\mathrm{dpz}=4)$. The ppfe parameter is instance-dependent (we only experimented with values 0,50 and 100).

\begin{tabular}{|c|c|c|c|c|c|c|c|c|}
\hline & BKS & ppfe & \#BKS & $\mathrm{APD}$ & $\mathrm{BPD}$ & WPD & Time & \#adopt \\
\hline els19 & 17212548 & 50 & 30 & 0 & 0 & 0 & 0.0 & 0.1 \\
\hline kra30a & 88900 & 100 & 30 & 0 & 0 & 0 & 0.0 & 0 \\
\hline sko56 & 34458 & 50 & 30 & 0 & 0 & 0 & 1.8 & 0.5 \\
\hline sko64 & 48498 & 50 & 30 & 0 & 0 & 0 & 2.0 & 0.3 \\
\hline sko72 & 66256 & 50 & 30 & 0 & 0 & 0 & 9.8 & 1.2 \\
\hline sko81 & 90998 & 50 & 30 & 0 & 0 & 0 & 22.4 & 1.6 \\
\hline sko90 & 115534 & 100 & 30 & 0 & 0 & 0 & 104.4 & 6.3 \\
\hline sko100a & 152002 & 100 & 27 & 0.001 & 0 & 0.016 & 129.3 & 3.4 \\
\hline sko100b & 153890 & 0 & 30 & 0 & 0 & 0 & 52.4 & 1.0 \\
\hline sko100c & 147862 & 0 & 30 & 0 & 0 & 0 & 77.5 & 1.3 \\
\hline sko100d & 149576 & 0 & 30 & 0 & 0 & 0 & 64.9 & 1.2 \\
\hline sko100e & 149150 & 0 & 30 & 0 & 0 & 0 & 49.4 & 0.9 \\
\hline sko100f & 149036 & 100 & 29 & 0.000 & 0 & 0.005 & 103.7 & 2.4 \\
\hline tai40a & 3139370 & 50 & 20 & 0.025 & 0 & 0.074 & 173.9 & 4.7 \\
\hline tai50a & 4938796 & 100 & 8 & 0.133 & 0 & 0.336 & 262.0 & 10.3 \\
\hline tai60a & 7205962 & 0 & 1 & 0.242 & 0 & 0.368 & 292.7 & 9.5 \\
\hline tai80a & 13499184 & 50 & 0 & 0.460 & 0.335 & 0.547 & 300.0 & 8.6 \\
\hline tai100a & 21052466 & 0 & 0 & 0.352 & 0.167 & 0.463 & 300.0 & 22.6 \\
\hline tai20b & 122455319 & 100 & 30 & 0 & 0 & 0 & 0.0 & 0.0 \\
\hline tai25b & 344355646 & 50 & 30 & 0 & 0 & 0 & 0.0 & 0.1 \\
\hline tai30b & 637117113 & 50 & 30 & 0 & 0 & 0 & 0.1 & 1.3 \\
\hline tai35b & 283315445 & 0 & 30 & 0 & 0 & 0 & 0.3 & 1.8 \\
\hline tai40b & 637250948 & 0 & 30 & 0 & 0 & 0 & 0.4 & 2.5 \\
\hline tai50b & 458821517 & 0 & 30 & 0 & 0 & 0 & 6.7 & 0 \\
\hline tai60b & 608215054 & 0 & 30 & 0 & 0 & 0 & 10.9 & 0 \\
\hline tai80b & 818415043 & 0 & 30 & 0 & 0 & 0 & 42.0 & 1.3 \\
\hline tai100b & 1185996137 & 0 & 29 & 0.001 & 0 & 0.024 & 143.4 & 4.9 \\
\hline tai150b & 498896643 & 50 & 0 & 0.190 & 0.085 & 0.410 & 300.0 & 10.1 \\
\hline tai64c & 1855928 & 0 & 30 & 0 & 0 & 0 & 0.2 & 0.1 \\
\hline tai256c & 44759294 & 50 & 0 & 0.264 & 0.211 & 0.312 & 300.0 & 4.4 \\
\hline tho40 & 240516 & 0 & 30 & 0 & 0 & 0 & 1.1 & 0.1 \\
\hline tho150 & 8133398 & 0 & 1 & 0.021 & 0 & 0.043 & 298.8 & 29.7 \\
\hline will 100 & 273038 & 100 & 26 & 0.000 & 0 & 0.002 & 144.7 & 5.2 \\
\hline Summary & & & 771 & 0.051 & 0.024 & 0.079 & 96.8 & 4.2 \\
\hline
\end{tabular}

Table 1: PHYSH-QAP on hard QAPLIB instances $(64$ cores, timeout=5 min $)$ 
Table 1 has all the results. For each instance we have the BKS, the ppfe parameter used, the number of times the BKS is reached (across the 30 executions), the Average Percentage Deviation (ADP) which is the average of the 30 relative deviation percentages computed as follows: $100 \times \frac{S o l-B K S}{B K S}$, the Best Percentage Deviation (BPD) which corresponds to the relative deviation percentage of the best solution found among the 30 executions, the Worst Percentage Deviation (WPD) which corresponds to the worst solution, the average execution time given in seconds which corresponds to the elapsed (wall) time, and includes the time to install all solver instances, solve the problem communications and the time to detect and propagate the termination and, finally, the average number of times the winning SU adopted an individual from the diverse/elite populations.

On this set of 33 hardest instances, even with a limit of time of 5 min PHYSHQAP is able to find the BKS at least once for 29 instances. Moreover, it is even able to reach the BKS systematically at each replication for 21 instances. For the 4 remaining instances (tai80a, tai100a, tai150b and tai256c), the quality of solutions returned by PHYSH-QAP is very good, around $0.2 \%$ of the BKS. The summary row has interesting numbers. The average ADP is only $0.051 \%$, the average BPD is $0.024 \%$ and the average WPD is $0.079 \%$. These numbers confirm that all runs provide high quality solutions; even the worst runs provide good results. For instance, in the worst case (tai80a), the worst solution among 30 runs is within just $0.547 \%$ of the BKS. Performance-wise, PHYSH-QAP averages just $96 \mathrm{sec}$ to find a solution. If we do not take into account the 4 unsolved instances (whose time is bounded by the time limit), the average run time is $70 \mathrm{sec}$. The number of adopted configurations on the wining $\mathrm{SU}$ is 4.2 , on average, showing that the hybridization is effectively taking place.

Comparison with another parallel hybrid solver for QAP: ParEOTS is a hybrid solver for QAP built on the top of the CPLS framework. ParEOTS combines RoTS and EO and has shown to perform very well. Indeed, on the hardest instances of QAPLIB, it outperforms most of state-of-the-art methods [11].

For this comparison we selected the 15 hardest instances from Table 1. We then ran ParEOTS using the parameters reported in [11] in the same execution environment as for PHYSH-QAP: same machine, using 64 cores with a time limit of $5 \mathrm{~min}$ and 30 repetitions per instance.

Table 2 presents the results. To compare the two solvers, compare the number of BKS found, then (in case of a tie), the APDs and finally the execution times. For each benchmark, the best-performing solver row is highlighted and the discriminant field is enhanced in bold font. PHYSH-QAP outperforms ParEOTS on 13 out of 15 of the hardest QAPLIB instances while the reverse only occurs for one instance (tai150b). Our implementation systematically solves 4 instances which are not fully solved on ParEOTS (sko81, sko90, sko100c and tai64c). The summary row shows that PHYSH-QAP obtains a total \#BKS higher than ParEOTS (232 vs. 195). It is worth noticing that this quality of solutions is obtained in a shorter execution time $(190 \mathrm{sec}$ vs. $208 \mathrm{sec})$. 


\begin{tabular}{lrrrrrrrr}
\hline & \multicolumn{3}{c}{ PHYSH-QAP } & & \multicolumn{3}{c}{ ParEOTS } \\
\cline { 2 - 3 } \cline { 7 - 8 } \cline { 6 - 8 } & \#BKS & APD & Time & & \#BKS & APD & Time \\
\hline sko81 & $\mathbf{3 0}$ & 0 & 22.4 & & 25 & 0.002 & 70.6 \\
sko90 & $\mathbf{3 0}$ & 0 & 104.4 & & 29 & 0.000 & 116.5 \\
sko100a & $\mathbf{2 7}$ & 0.001 & 129.3 & & 25 & 0.003 & 128.9 \\
sko100c & $\mathbf{3 0}$ & 0 & 77.5 & & 29 & 0.000 & 127.3 \\
tai40a & 20 & 0.025 & $\mathbf{1 7 3 . 9}$ & & 20 & 0.025 & 184.2 \\
tai50a & $\mathbf{8}$ & 0.133 & 262.0 & & 3 & 0.144 & 289.8 \\
tai60a & $\mathbf{1}$ & 0.242 & 292.7 & & 0 & 0.270 & 300.0 \\
tai80a & 0 & 0.460 & 300 & & 0 & 0.460 & 300.0 \\
tai100a & 0 & $\mathbf{0 . 3 5 2}$ & 300 & & 0 & 0.358 & 300.0 \\
tai100b & $\mathbf{2 9}$ & 0.001 & 143.4 & & 22 & 0.015 & 181.4 \\
tai150b & 0 & 0.190 & 300.0 & & 0 & $\mathbf{0 . 1 3 0}$ & 300.0 \\
tai64c & $\mathbf{3 0}$ & 0 & 0.2 & & 28 & 0.004 & 20.0 \\
tai256c & 0 & $\mathbf{0 . 2 6 4}$ & 300.0 & & 0 & 0.272 & 300.0 \\
tho150 & $\mathbf{1}$ & 0.021 & 298.8 & & 0 & 0.019 & 300.0 \\
wil100 & $\mathbf{2 6}$ & 0 & 144.7 & & 14 & 0.001 & 213.9 \\
\hline Summary & $\mathbf{2 3 2}$ & $\mathbf{0 . 1 1 3}$ & $\mathbf{1 9 0 . 0}$ & & 195 & 0.114 & 208.8 \\
\hline
\end{tabular}

Table 2: PHYSH-QAP vs ParEOTS (64 cores, timeout=5 min)

\subsection{Evaluation of PHYSH-QAP on harder instances}

We evaluated our hybrid solver on two sets of instances, artificially crafted to be very difficult for metaheuristics: the dre $X X$ instances proposed by Drezner at al. 6] and the Inst $X X$ instances by Palubeckis [17. These instances are generated with a known optimum. For this test we used the same machine, with 128 cores and a time limit of $10 \mathrm{~min}$ with 30 repetitions. We used the same framework configuration as in Section 4.1 for QAPLIB. We could not yet experiment with different values for ppfe so we use ppfe $=100$ for all instances.

\begin{tabular}{lrrrr}
\hline & \#BKS & APD & best & Time \\
\hline dre21 & $\mathbf{3 0}$ & 0 & $\mathbf{3 5 6}$ & 0.0 \\
dre24 & $\mathbf{3 0}$ & 0 & $\mathbf{3 9 6}$ & 0.0 \\
dre28 & $\mathbf{3 0}$ & 0 & $\mathbf{4 7 6}$ & 0.0 \\
dre30 & $\mathbf{3 0}$ & 0 & $\mathbf{5 0 8}$ & 0.1 \\
dre42 & $\mathbf{3 0}$ & 0 & $\mathbf{7 6 4}$ & 0.9 \\
dre56 & $\mathbf{3 0}$ & 0 & $\mathbf{1 0 8 6}$ & 11.5 \\
dre72 & $\mathbf{3 0}$ & 0 & $\mathbf{1 4 5 2}$ & 90.9 \\
dre90 & $\mathbf{2 3}$ & 2.757 & $\mathbf{1 8 3 8}$ & 281.2 \\
dre110 & $\mathbf{6}$ & 14.997 & $\mathbf{2 2 6 4}$ & 549.4 \\
dre132 & $\mathbf{5}$ & 11.404 & $\mathbf{2 7 4 4}$ & 558.2 \\
\hline Summary & 244 & 2.915 & & 149.2 \\
\hline
\end{tabular}

\begin{tabular}{lrrrr}
\hline & \#BKS & APD & \multicolumn{1}{c}{ best } & Time \\
\hline Inst20 & $\mathbf{3 0}$ & 0 & $\mathbf{8 1 5 3 6}$ & 0.0 \\
Inst30 & $\mathbf{3 0}$ & 0 & $\mathbf{2 7 1 0 9 2}$ & 0.1 \\
Inst40 & $\mathbf{3 0}$ & 0 & $\mathbf{8 3 7 9 0 0}$ & 3.2 \\
Inst50 & $\mathbf{3 0}$ & 0 & $\mathbf{1 8 4 0 3 5 6}$ & 7.7 \\
Inst60 & $\mathbf{3 0}$ & 0 & $\mathbf{2 9 6 7 4 6 4}$ & 11.8 \\
Inst70 & $\mathbf{3 0}$ & 0 & $\mathbf{5 8 1 5 2 9 0}$ & 35.7 \\
Inst80 & $\mathbf{3 0}$ & 0 & $\mathbf{6 5 9 7 9 6 6}$ & 78.0 \\
Inst100 & $\mathbf{1 7}$ & 0.038 & $\mathbf{1 5 0 0 8 9 9 4}$ & 476.4 \\
Inst150 & 0 & 0.122 & $\mathbf{5 8 4 1 1 4 8 4}$ & 600.0 \\
Inst200 & 0 & 0.123 & $\mathbf{7 5 4 9 5 9 6 0}$ & 600.0 \\
\hline Summary & 227 & 0.028 & & 181.3 \\
\hline
\end{tabular}

Table 3: PHYSH-QAP on Drezner and Palubeckis (128 cores, timeout=10 min) 
Table 3 presents the results obtained on both benchmarks. Regarding Drezner's instances, PHYSH-QAP is able to optimally solve all instances. To best of our knowledge, no other dedicated solver for QAP has ever reported an optimal solution either for dre110 or dre132 (highlighted in green in the table). Moreover, all instances of size $n \leq 72$ are systematically solved at each replication. Regarding Palubeckis' instances, the optimum is found for instances with $n \leq 100$ (and systematically found at each replication for $n \leq 80$ ). For size $n>100$, clearly a limit of $10 \mathrm{~min}$ is too short. Nevertheless the quality of obtained solutions within this time limit is very good with an APD around $0.12 \%$. It is worth noting that for Inst150 and Inst200, the solution computed by PHYSH-QAP improves on the best solutions ever published (corresponding best costs computed are highlighted in green in Table 3 .

\section{Conclusion and Future Directions}

We have proposed PHYSH: a new framework for the efficient resolution of Combinatorial Optimization Problems combining single-solution metaheuristics, population-based metaheuristics, cooperative parallelism and hybridization. We have used our X10 implementation of this framework to construct a hybrid solver for the Quadratic Assignment Problem which combines up to three metaheuristics. This solver turns out to perform exceptionally well, particularly on very hard instances of QAP.

We plan to study the impact of each parameter in more detail; including experimentation with techniques for parameter auto-tuning, e.g. using F-Race. We also plan to add new metaheuristics to the prototype, particularly populationbased methods. This enriched implementation we will enable uas to address a wider range of problems. Finally, it will be interesting to experiment on different parallel architectures, for instance GPGPUs or Intel MIC, using the X10 language, which greatly abstracts on machine architectural specificities.

\section{References}

1. Blum, C., Roli, A.: Metaheuristics in Combinatorial Optimization: Overview and Conceptual Comparison. ACM Computing Surveys 35(3), 268-308 (2003)

2. Burkard, R.E., Karisch, S., Rendl, F.: QAPLIB - a Quadratic Assignment Problem Library. European Journal of Operational Research 55(1), 115-119 (1991)

3. Caniou, Y., Codognet, P., Richoux, F., Diaz, D., Abreu, S.: Large-scale Parallelism for Constraint-Based Local Search: the Costas Array Case Study. Constraints 20(1), 30-56 (2015)

4. Crainic, T., Gendreau, M., Hansen, P., Mladenovic, N.: Cooperative Parallel Variable Neighborhood Search for the p-Median. Journal of Heuristics 10(3), 293-314 (2004)

5. Crainic, T., Toulouse, M.: Parallel Meta-Heuristics. In: Gendreau, M., Potvin, J.Y. (eds.) Handbook of Metaheuristics, $\{\mathrm{I}\}$ nternational $\{\mathrm{S}\}$ eries in $\{\mathrm{O}\}$ perations $\{R\}$ esearch \& $\{\mathrm{M}\}$ anagement $\{\mathrm{S}\}$ cience, vol. 146, pp. 497-541. Springer US (2010) 
6. Drezner, Z.: The Extended Concentric Tabu for the Quadratic Assignment Problem. European Journal of Operational Research 160(2), 416-422 (2005)

7. Drezner, Z.: Extensive experiments with hybrid genetic algorithms for the solution of the quadratic assignment problem. Computers \& Operations Research 35(3), 717-736 (2008)

8. Hoos, H., Stützle, T.: Stochastic Local Search: Foundations and Applications. Morgan Kaufmann / Elsevier (2004)

9. Misevicius, A.: A Tabu Search Algorithm for the Quadratic Assignment Problem. Computational Optimization and Applications 30(1), 95-111 (jan 2005)

10. Moscato, P., Cotta, C.: Memetic algorithms. Handbook of Applied Optimization 157, $168(2002)$

11. Munera, D., Diaz, D., Abreu, S.: Hybridization as Cooperative Parallelism for the Quadratic Assignment Problem. In: 10th International Workshop, HM 2016. Lecture Notes in Computer Science, vol. 9668, pp. 47-61. Springer International Publishing, Plymouth, UK (2016)

12. Munera, D., Diaz, D., Abreu, S.: Solving the Quadratic Assignment Problem with Cooperative Parallel Extremal Optimization. In: The 16th European Conference on Evolutionary Computation in Combinatorial Optimisation. Porto (2016)

13. Munera, D., Diaz, D., Abreu, S., Codognet, P.: A Parametric Framework for Cooperative Parallel Local Search. In: Blum, C., Ochoa, G. (eds.) European Conference on Evolutionary Computation in Combinatorial Optimisation (EvoCOP). Lecture Notes in Computer Science, vol. 8600, pp. 13-24. Springer, Granada, Spain (2014)

14. Munera, D., Diaz, D., Abreu, S., Codognet, P.: Flexible Cooperation in Parallel Local Search. In: Symposium on Applied Computing, SAC'2014. pp. 1360-1361. ACM Press, Gyeongju, Korea (2014)

15. Munera, D., Diaz, D., Abreu, S., Rossi, F., Saraswat, V., Codognet, P.: Solving Hard Stable Matching Problems via Local Search and Cooperative Parallelization. In: AAAI. Austin, TX, USA (2015)

16. Novoa, C., Qasem, A., Chaparala, A.: A SIMD tabu search implementation for solving the quadratic assignment problem with GPU acceleration. Proceedings of the 2015 XSEDE Conference on Scientific Advancements Enabled by Enhanced Cyberinfrastructure - XSEDE '15 pp. 1-8 (2015)

17. Palubeckis, G.: An Algorithm for Construction of Test Cases for the Quadratic Assignment Problem. Informatica, Lith. Acad. Sci. 11(3), 281-296 (2000)

18. Saifullah Hussin, M.: Stochastic Local Search Algorithms for Single and Biobjective Quadratic Assignment Problems. Ph.D. thesis, Université de Bruxelles (2016)

19. Taillard, É.: Robust Taboo Search for the Quadratic Assignment Problem. Parallel computing 17(4-5), 443-455 (1991)

20. Talbi, E.G., Bachelet, V.: COSEARCH: A parallel cooperative metaheuristic. Journal of Mathematical Modelling and Algorithms 5(1), 5-22 (2006)

21. Toulouse, M., Crainic, T., Gendreau, M.: Communication Issues in Designing Cooperative Multi-Thread Parallel Searches. In: Osman, I., Kelly, J. (eds.) MetaHeuristics: Theory\&Applications, pp. 501-522. Kluwer Academic Publishers, Norwell, MA. (1995)

22. Tsutsui, S., Fujimoto, N.: An Analytical Study of Parallel GA with Independent Runs on GPUs, vol. 8 (2013) 